\title{
Visualization of Flows around a Wide Au-Coated Beam in Valving Motion Understood as AC Electroosmosis
}

\author{
Hideyuki Sugioka* and Yuki Mizuno \\ Department of Mechanical Systems Engineering, \\ Shinshu University 4-17-1 Wakasato, Nagano 380-8553, Japan
}

(Dated: February 28, 2020)

\begin{abstract}
Recently, bio-inspired devices have attracted much attention. In particular, elastic valves using AC electroosmosis (ACEO) hold promise for the development of artificial cilia in future microfluidic applications because of their simplicity and high-performance multi-functionality. However, direct evidence of ACEO-driven large valving motion has not been obtained yet. In this study, we report experiments that a wide Au-coated plastic-film beam shows large valving motion in water. By visualizing the flow fields during the motion, we show that the large valving motion of the wide Au-coated beam is understood to be due to ACEO occurring at the edge of the beam. Furthermore, we found that the wide beam was elevated largely by the effect of the vortex flow (understood to be the flow due to ACEO at the beam edge) and the incoming lift flow into the rearward region of the valve. We believe that our findings contribute significantly to realizing new artificial cilia.
\end{abstract}

PACS numbers: 47.57.-s, 85.90.+h, 82.45.Hk, 83.50.Lh

*hsugioka@shinshu-u.ac.jp 


\section{INTRODUCTION}

Bio-inspired machines suggest the existence of many applications that can be implemented using technology and leading-edge physics. In particular, artificial cilia [1] have been attracting much attention, since they may provide ideal multifunctional microfluidic devices for Lab-on-a-chip and micro-total analytical systems ( $\mu$ TAS) [2]. Hanasoge et al. demonstrated microfluidic pumping corresponding to a centerline velocity equal to $\sim 1.35$ mm/s using a magnetic NiFe film driven by external magnetic field [3]. Panigrahi et al. demonstrated a mixing function for sperm activation using magnetic cilia made of a polymer incorporating neodymium-iron-boron particles [4]. Asadnia et al. demonstrated a highsensitivity flow sensor using a ciliary bundle linked with a piezoelectric nanofiber [5]. Mani et al. demonstrated a zebrafish's orientation being manipulated by using artificial cilia fabricated from shape-memory alloy [6]. However, magnetic cilia, which are the most popular form, need a large magnet and, thus, miniaturization is inherently difficult.

Conversely, electrostatic cilia that are driven by an electric field have a significant advantage for miniaturization. For example, Toonder et al. demonstrated net fluid flow and mixing in silicon oil using curled micro-metal beam electrostatic cilia that are driven by AC electric fields [7]. However, as pointed out by Toonder and Onck, the requirement of silicone oil of Toonder et al.'s cilia is considered a serious impediment to biomedical applications citetoon1. For this problem, we theoretically showed that electrostatic cilia using $\mathrm{AC}$ electroosmosis (ACEO) works in water with various attractive microfluidic functions, i.e., valving [8], pumping [9], and catching [10]. Furthermore, we experimentally proved the fundamental motion of ACEO elastic cilia made of a narrow plate-type gold leaf in water [11]. Further, we recently demonstrated stable periodic beating motion of ACEO elastic cilia fabricated from spiral gold thread with a two-channel (2-Ch) electrode structure [12]. Note that ACEO [13] [more generally, induced charge electroosmosis [14]] is a nonlinear electrokinetic phenomenon that produces a strong flow proportional to the square of the applied electric field. Further, ACEO cilia are suitable for miniaturization of the entire apparatus Theoretically, they move in a typical aqueous microfluidic channel with the width of $\sim 200$ $\mu \mathrm{m}$ and high ACEO flow speed of $\sim 1 \mathrm{~mm} / \mathrm{s}$ at low applied voltages of $\sim 1 \mathrm{~V}$ [8]. Therefore, we consider that they are promising to be the next-generation microfluidic devices.

However, large motion of a wide conductive elastic-plate beam due to ACEO has not been 
proven experimentally yet because the wide, gold leaf simple-plate-beam is too fragile to fabricate. Further, the flow fields around the ACEO elastic actuator have not been observed and thus the evidence that the mechanism is due to ACEO is not sufficient yet, although it is proven that the deviation of the ACEO elastic actuator is proportional to the square of the applied electric field, which suggests that the beating motion occurs due to ACEO flows. Note that theoretically the net pumping flow of the ACEO elastic actuator originates from the ACEO downflow (of the beam edge during the application of $\mathrm{AC}$ voltage), which impels the forward flow [9]. That is, unlike that of other cilia, the net pumping flow of the ACEO cilia is produced by the asymmetrical flow between the up-wing and down-wing motions of the elastic beam. Consequently, if the motion of the beam is not due to an ACEO flow but due to an external force (such as dielectrophoretic force or Maxwell stress), the periodic beating motion would not produce net flows in the low Reynolds (Re) regime. Therefore, it must be established that the motion of our actuators elastic beam is due to the ACEO flows.

In this study, we report that under AC electric fields, a wide Au-coated plastic-film beam demonstrates large valving motion in water, leading us to conclude that it occurs due to the ACEO flows. By directly observing the flow fields around the ACEO cilia, we explain the large valving motion mechanism.

\section{METHOD}

Fig. 1 shows a schematic of the experimental setup for observing the valving motion of a wide Au-coated plastic-film beam due to AC electro-osmotic flows. The side view is shown in Fig. 1(a). The top view is shown in Fig. 1(b). As shown in Fig. 1 (a), we set the Au-coated plastic-film beam of width $W(=9 \mathrm{~mm})$, length $L(=15 \mathrm{~mm})$, and thickness $d(=0.01 \mathrm{~mm})$ on the lower (left) $\mathrm{Cu}$ electrode and placed the upper $\mathrm{Cu}$ electrode of length $L^{\prime}(=20 \mathrm{~mm})$ opposite the beam. The beam was fabricated from polyethylene (PE) film, and for conductivity, it was coated with an Au film of $100 \mathrm{~nm}$ thick. The initial edge position $A$ of the beam is $\left(0, h_{0}\right)$, the temporary edge position $B$ during the valving motion is $\left(x_{B}, y_{B}\right)\left[=\left(x_{B}, h_{0}+h\right)\right]$, and the final edge position is approximately $\left(x_{f}, h_{0}+h_{f}\right)$, where $x_{B}$ and $x_{f}$ are the temporary and final $x$ positions of the beam edge, respectively. Here, the origin of the coordinates is the point $\mathrm{O}$ directly below the point $\mathrm{A}$ on the surface of the 

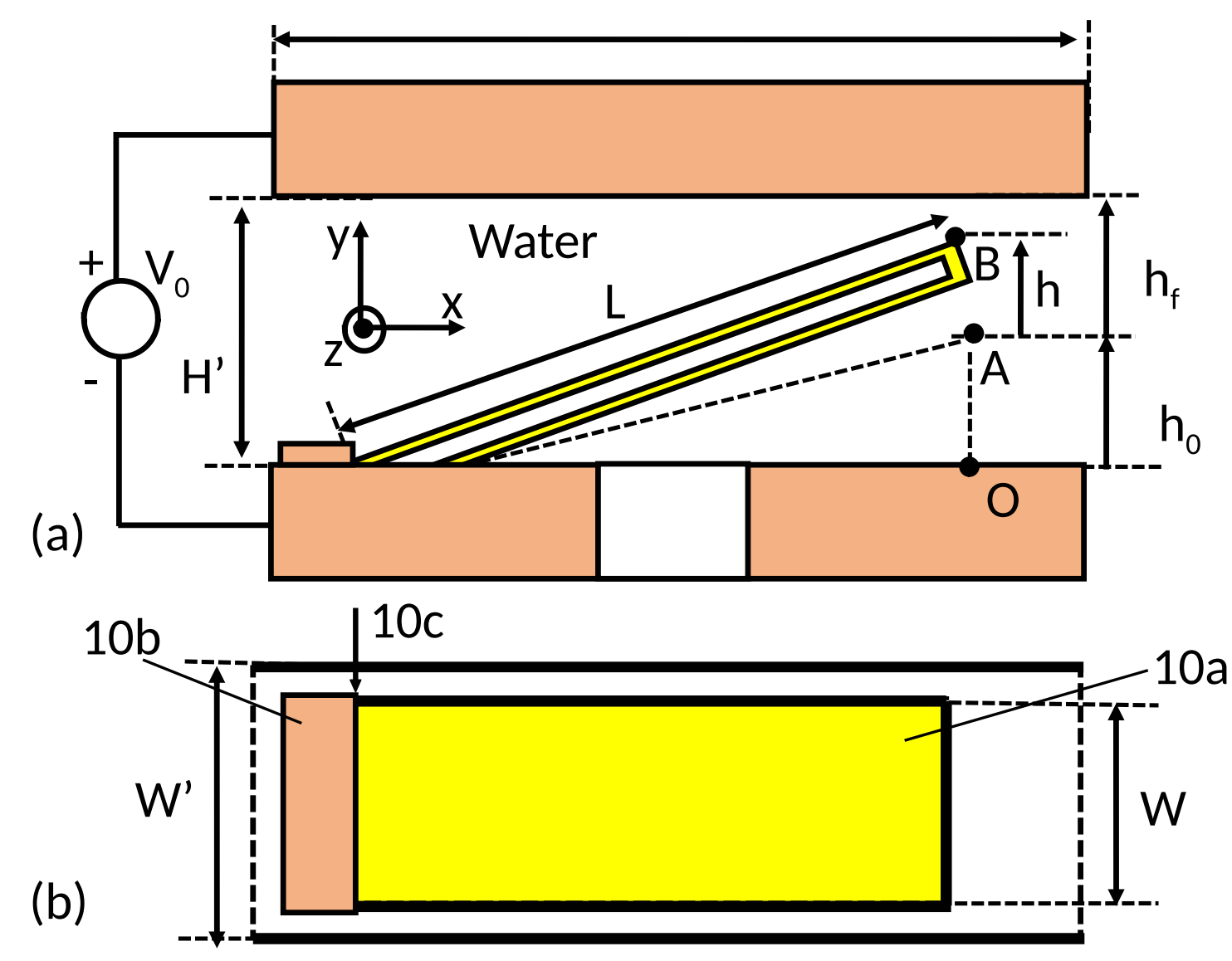

FIG. 1. (Color online) Experimental setup for observing the valving motion of a wide Au-coated plastic-film beam due to AC electro-osmotic flows. (a) Side view. (b) Top view. An Au-coated plastic-film beam $W(=9 \mathrm{~mm})$ wide, $L(=15 \mathrm{~mm})$ long, and $d(=0.01 \mathrm{~mm})$ thick; $H^{\prime}=4 \mathrm{~mm}$, $W^{\prime}=11 \mathrm{~mm}$, and $L^{\prime}=20 \mathrm{~mm}$. 10a: Au-coated plastic-film beam, 10b: Cu tape beam stopper, 10c: $\mathrm{Cu}$ tape position to fix the beam.

lower electrode. Furthermore, as shown in Fig. 1(b), the beam was placed in a channel with the width of $W^{\prime}(=11 \mathrm{~mm})$ and height of $H^{\prime}(=4 \mathrm{~mm})$. The space bounded by the walls and the electrodes is the valving motion chamber. The lower edge of the beam was fixed by using $\mathrm{Cu}$ tape [denoted by $10 \mathrm{~b}$ in Fig. 1(b)]. After injecting water (milli-Q, $18.2 \mathrm{M} \Omega \mathrm{cm}$ initially), which included a pearl pigment tracer, into the chamber, we applied a rectangular AC voltage of peak value $V_{0}$ and frequency $f$ between the upper and lower electrodes and observed the motion of the beam. Here, we repeated the observation $N_{f}$ times (typically, $N_{f}=3$ ) by changing $f$ and $V_{0}$. By using recorded video data with the size of $1280 \times 720$ and 
a frame rate of $2425 \mathrm{fps}$, we determined the temporary edge position $\left(x_{B}(t), y_{B}(t)\right)$ with the flow fields $\boldsymbol{u}=\left(u_{x}(x, y, t), u_{y}(x, y, t)\right)$, where $t$ is time. We defined $t=0$ as the AC voltage application start time Thus, $h(0)=0$ and $h_{0}$ show the initial conditions of the system. We defined $U_{y}$ as the maximum flow velocity in the $y$ direction in the region $\mathrm{D}=\{(x, y) \mid 0 \leq x \leq 1$ $\mathrm{mm}, 0 \leq y \leq 4 \mathrm{~mm}\}$; i.e.,

$$
U_{y}(t)=\max _{(x, y) \in D} u_{y}(x, y, t)
$$

The chamber was fabricated from polylactic acid (PLA) using a 3D printer, and we used 1 $\mathrm{k} \Omega$ resistance to protect the circuit. The lower electrode was divided into two parts, namely, left and right, for future 2-Ch driving experiment [12], as shown in Fig. 1(a), although the right electrode was not used presently.

\section{RESULTS}

\section{A. Motion when the beam stopped during the up-wing motion}

We observed that the beam occasionally stopped during the up-wing motion. Figs. 2(a) and 2(b) show the dependences of $h$ and $v_{b} \equiv \frac{d h}{d t}$, respectively, on $t$ at $V_{0}=40 \mathrm{~V}$ and $f=100 \mathrm{~Hz}$. Here, three independent measurements (i.e., $N_{f}=3$ ) were performed under the same conditions. In Figs. 2(a) and 2(b), we found that the edge velocity $v_{b}$ of this device tended to drop significantly at $t \simeq 1 \mathrm{~s}$ and $h \simeq 1 \mathrm{~mm}$. We occasionally observed a pausing period (e.g., approximately $6 \mathrm{~s}$ for $N=1$ ) during the up-wing motion of the beam. Figs. 2(c) and 2(d) show the dependence of $h_{f} / T$ on $f$ and $V_{0}$, respectively, where $T$ is the time taken to reach the steady state and $h_{f} / T$ represents the average velocity of the up-wing motion of the edge. As shown in Fig. $2(\mathrm{c}), h_{f} / T$ has the peak value at $f \sim 5 \mathrm{kHz}$, and this frequency characteristic approximately agrees with that of ordinary ACEO pumps [15, 16]. We consider that this narrow band characteristic is due to the two-dimensional structure of the wide beam, and it significantly differs from the broadband characteristic of the elastic actuator having a skew structure [11]. Furthermore, as shown in Fig. 2(d), the average velocity is proportional to the square of $V_{0}$ corresponding to the nonlinear characteristics of ACEO. 


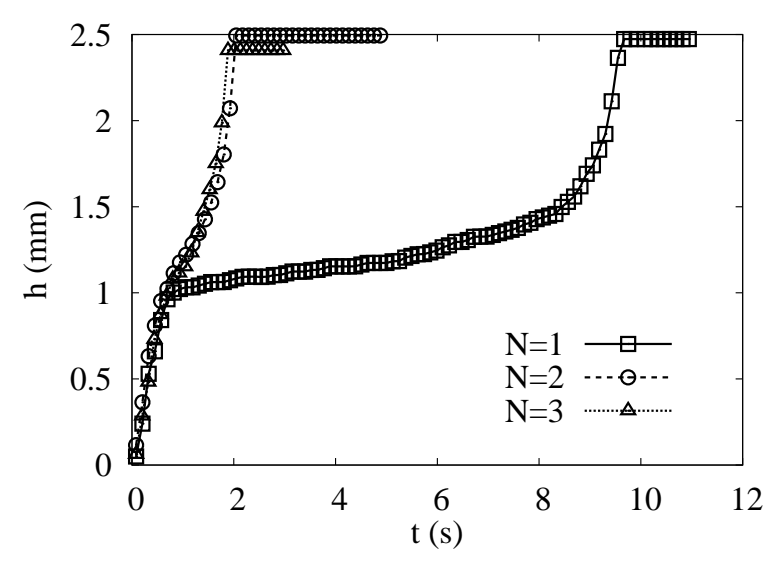

(a) Dependence of $h$ on $t$

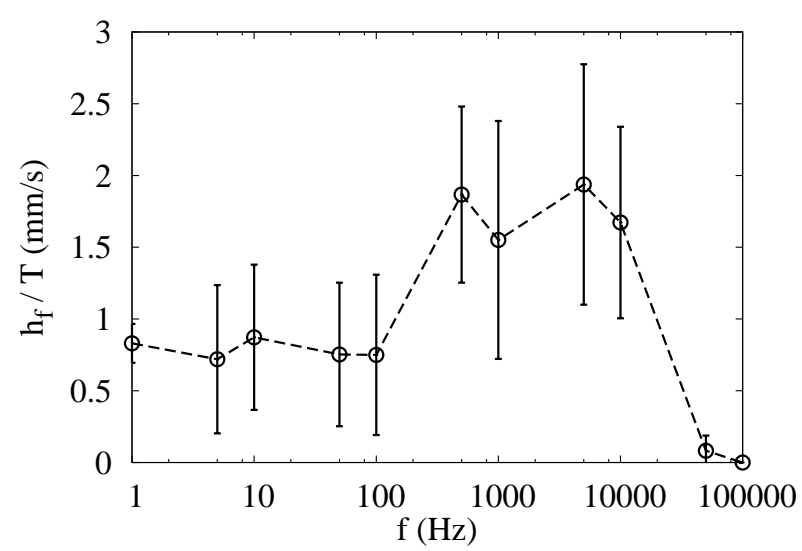

(c) Dependence of $h_{f} / T$ on $f$ at $V_{0}=40 \mathrm{~V}$

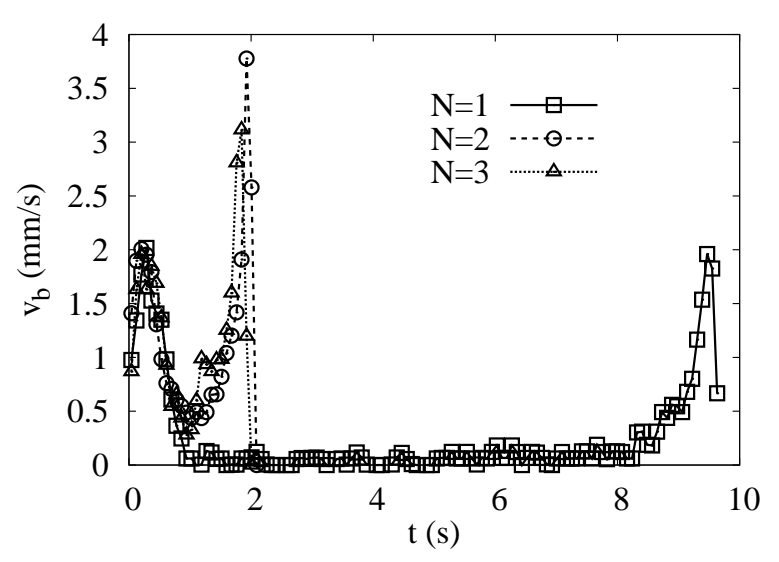

(b) Dependence of $v_{b}$ on $t$

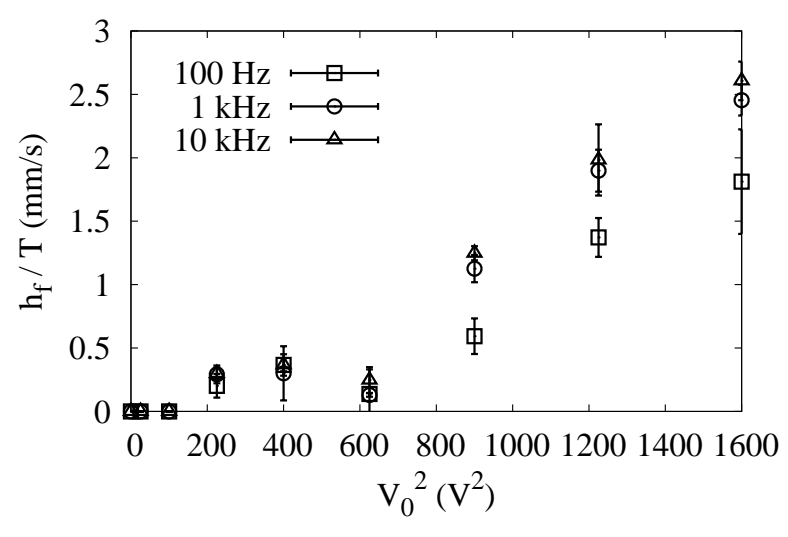

(d) Dependence of $h_{f} / T$ on $V_{0}^{2}$

FIG. 2. Characteristics of the valving motion when the beam stopped during the up-wing motion. In (a) and (b), $V_{0}=40 \mathrm{~V}, f=100 \mathrm{~Hz}$, and $h_{f}=2.23$ to $2.49 \mathrm{~mm}\left(h_{0}=1.51\right.$ to $\left.1.77 \mathrm{~mm}\right)$.

\section{B. Visualization of the flow fields when the beam stopped during the up-wing}

\section{motion}

Figs. 3(a), 3(b), 3(c), and 3(d) show the photographs of the typical valving motion (including a pausing period) at $t=0.04,1.29,3.22$, and $8.88 \mathrm{~s}$ under an $\mathrm{AC}$ electric field. Here, $V_{0}=40 \mathrm{~V}, f=100 \mathrm{~Hz}$, and $h_{f}=2.47 \mathrm{~mm}$. As shown in Fig. 3(a), the beam started to move in the upper direction with a relatively small clockwise vortex flow near the edge of the beam . Gradually, the vortex flow velocity increased as shown in Fig. 3(b). Further, when the beam motion was stopped, we observed that upper and lower vortex flows continued, as shown in Fig. 3(c). We also observed that the wide beam was elevated remarkably by the actions of the upper vortex flow near the beam edge and the incoming lift flow into the 

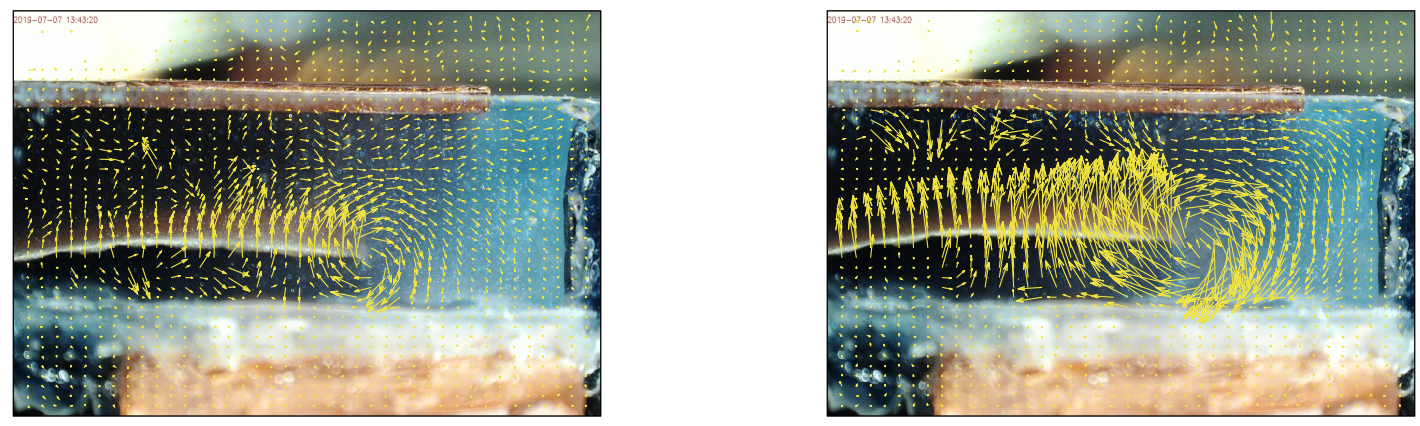

(a) $t=0.04 \mathrm{~s}$

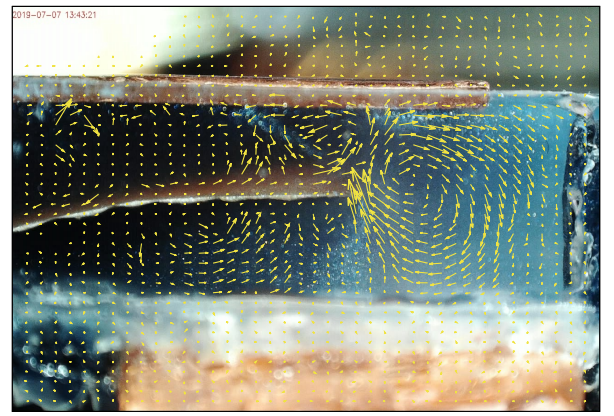

(b) $t=1.29 \mathrm{~s}$

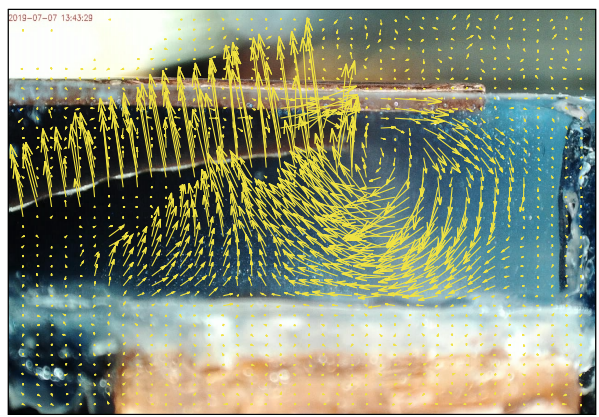

(c) $t=3.22 \mathrm{~s}$

(d) $t=8.88 \mathrm{~s}$

FIG. 3. (Color online) Photographs of the valving motion that accompanies a pause under an AC electric field with the flow fields around the beam. Here, $N=1, V_{0}=40 \mathrm{~V}, f=100 \mathrm{~Hz}, h_{0}=1.53$ $\mathrm{mm}$, and $h_{f}=2.47 \mathrm{~mm} ; f_{a}=1$ is a relative magnification factor of the flow vector.

bottom region of the valve (the flow resulted from the continuous valving motion due to the upper vortex flow), as shown in Fig. 3(d).

\section{Flow fields stopped during the up-wing motion by an obstacle (toothpick)}

Fig. 4 shows the experimental results using a real obstacle. Here, we used a toothpick to stop the motion of the beam. Specifically, Figs. 4(a), 4(b), and 4(c) show the flow fields at $t=0.41,0.66$, and $6.72 \mathrm{~s}$, respectively. As shown in Fig. 4(a), we observed the clockwise vortex flow immediately before the beam was stopped. Then, we observed an inertial flow immediately after the beam was stopped, as shown in Fig. 4(b). After the inertial flow 
disappeared ( $\sim 0.2 \mathrm{~s}$ from the video data), we observed upper and lower edge vortex flows, as shown in Fig. 4(c). That is, the experimental results using a real obstacle are the same as the results of beam stoppage during the up-wing motion (in Figs. 2 and 3). Note that Fig. 4(d) shows the dependence of $h$ on $t$ for this experiment.

\section{Visualization of flow field of a periodic valving motion}

To ensure the applicability of this elastic actuator to artificial cilia, we demonstrated the periodic valving motion under conditions that $V_{0}=40 \mathrm{~V}, f=50 \mathrm{~Hz}, f_{b}=0.5 \mathrm{~Hz}$,

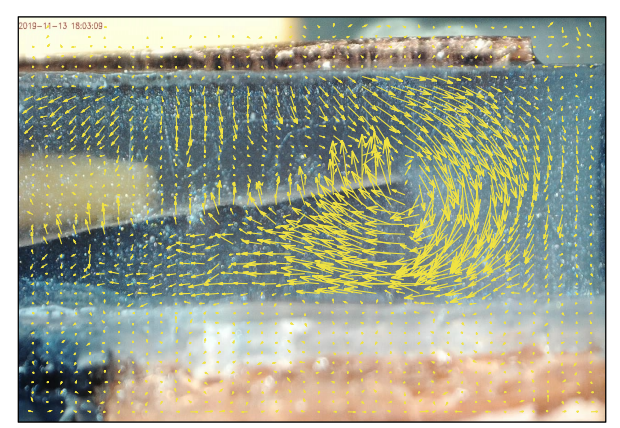

(a) $t=0.41 \mathrm{~s}$ (immediately before the beam is stopped)

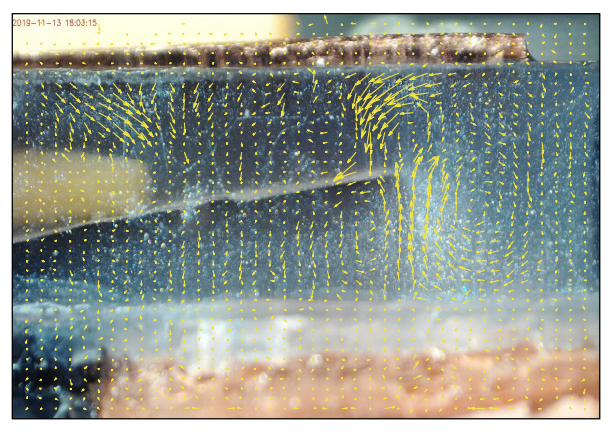

(c) $t=6.72 \mathrm{~s}$

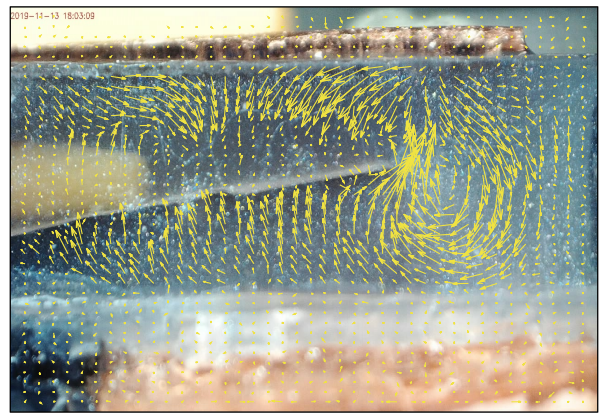

(b) $t=0.66 \mathrm{~s}$ (immediately after the beam is stopped)

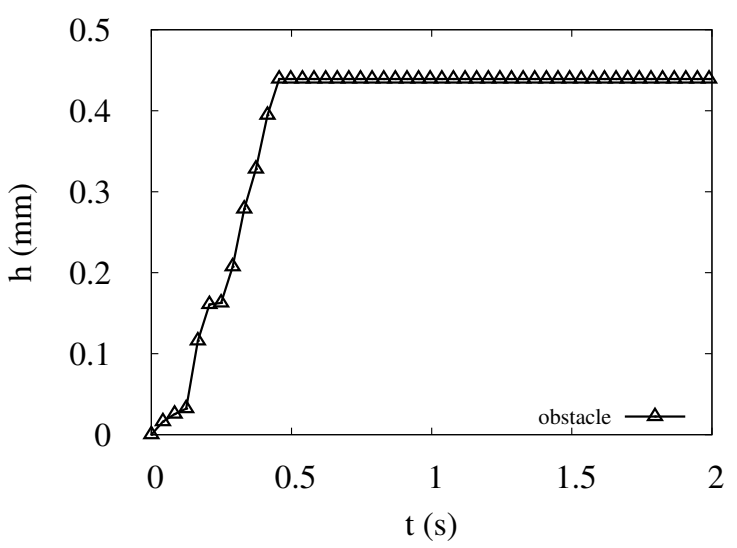

(d) Dependence of $h$ on $t$

FIG. 4. (Color online) Experimental results using a real obstacle (toothpick). Here, $N=2$, $V_{0}=40 \mathrm{~V}, f=100 \mathrm{~Hz}, h_{0}=1.43 \mathrm{~mm}$, and $h_{f}=2.57 \mathrm{~mm} ; f_{a}=2$ is a relative magnification factor of the flow vector. 
and $T_{b}=2 \mathrm{~s}$ in Fig. 5, where $f_{b}$ and $T_{b}$ are the frequency and period, respectively, of the valving motion. That is, an $\mathrm{AC}$ voltage is applied at $0 \leq t \leq \frac{T_{b}}{2}$ and no voltage is applied at $\frac{T_{b}}{2} \leq t \leq T_{b}$ for one period. Specifically, Figs. 5(a) and 5(b) show the photographs of up-wing and down-wing motions, respectively, of our device. Here, the up-wing motion resulted from the edge downflow determined to be ACEO at the edge (as will be discussed in Sec. IV), whereas the down-wing motion resulted from the elastic force of the elastic beam. Furthermore, Figs. 5(c) and 5(d) show the dependences of $h$ and $U_{y}$ on $t$. In Fig. 5(c), we can observe a periodic valving (beating) motion; thus, we can use our actuator as artificial cilia, although the beam is not completely returned to the initial position; i.e., we need to use a longer rest period (or a lower $f_{b}$ ) for a complete recovery. Note that the beam did not move immediately after switching off the applied voltage as shown in Fig. 5(c), although

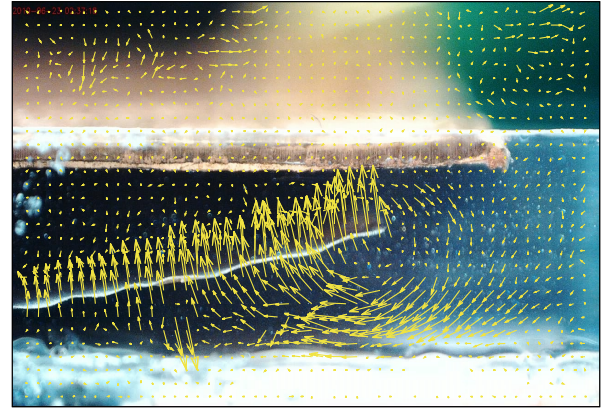

(a) Up-wing motion $(t=0.205 \mathrm{~s})$

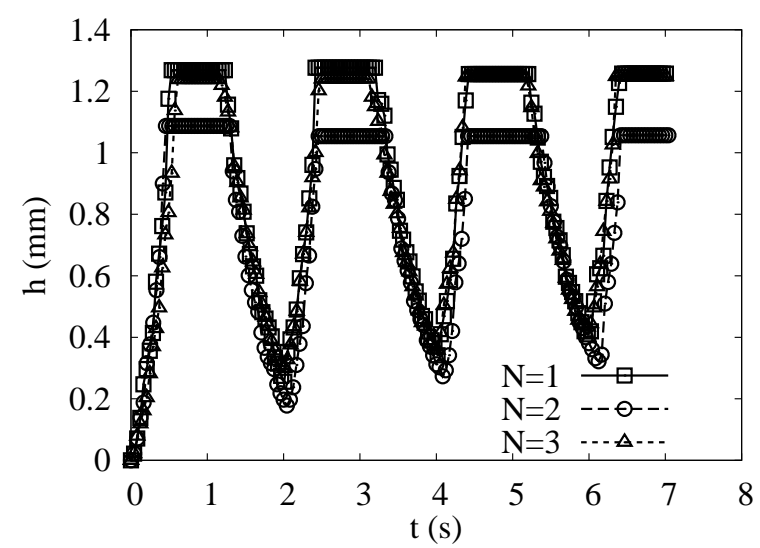

(c) Dependence of $h$ on $t$

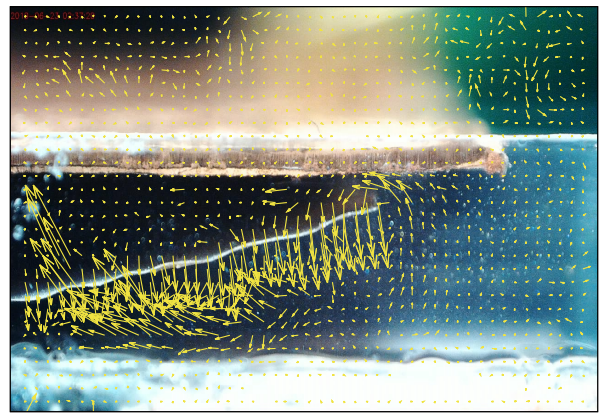

(b) Down-wing motion $(t=1.64 \mathrm{~s})$

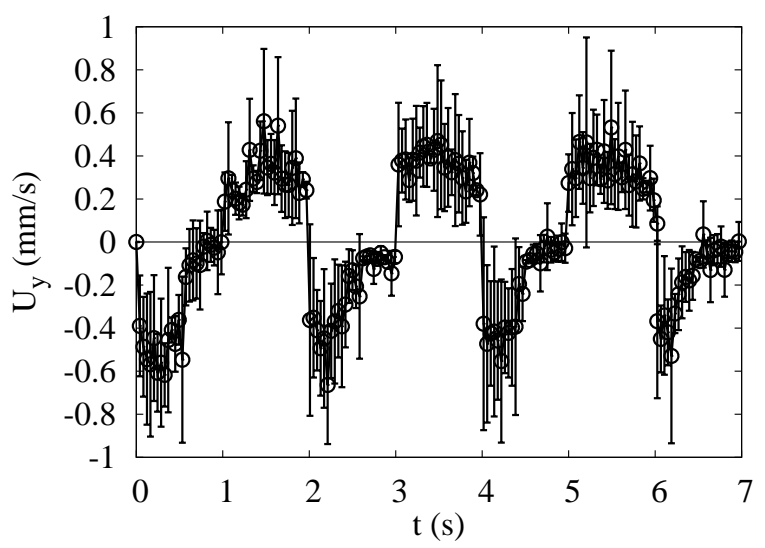

(d) Dependence of $U_{y}$ on $t$

FIG. 5. (Color online) Periodic valving motion. Here, $V_{0}=40 \mathrm{~V}, f=50 \mathrm{~Hz}, f_{b}=0.5 \mathrm{~Hz}$, and $h_{f}=1.25$ to $1.27 \mathrm{~mm} ; f_{a}=1$ is a relative magnification factor of the flow vector. 
the sign of $U_{y}$ changed from negative to positive as shown in Fig. 5(d). This was probably because the amount of deflection changed without changing the edge position at $1 \leq t<1.2$ s. Furthermore, although the flat region in Fig. 5(c) shows that the beam edge reached to the upper electrode, we find that $U_{y}$ was not zero at $t<\frac{T_{b}}{2}$ and $U_{y}$ was still negative. This is probably because the beam edge was in contact with the upper electrode through the insulating pearl pigment; i.e., $V_{0}$ continued to be applied between the beam and the upper electrode, and thus the edge flow (taken as the flow due to ACEO) continued to flow after the contact.

\section{DISCUSSION}

\section{A. Cause to stop during the up-wing motion}

Although the ideal shape of the beam cross-section is rectangular, we consider that there are slight distortions in the $z$ direction and that the cross-section sometimes changes from convex upward [in Fig. 6(a)] to convex downward [in Fig. 6(b)] at $t \simeq 1 \mathrm{~s}$ when the edge of the beam moves in the $y$ direction. Thus, in Fig. 2(b), $v_{b}$ decreased significantly at $t \simeq 1 \mathrm{~s}$ to change the cross-section from convex to concave. That is, the shape transformation from convex to concave functioned as an obstacle. Thus, we call it a "conceptual obstacle" and consider that the beam at $N=1$ is almost stopped by this conceptual obstacle during $t \simeq 1$ to $8 \mathrm{~s}$ as shown in Fig. 2(b). In fact, the experimental results using a real obstacle in Fig. 4 are similar. Figs. 6(a) and 6(b) are the conceptual figures of the cross-sectional shape of the beam by estimation.

\section{B. Interpretation of the flow fields around the beam}

From the direct observation of the flow field, we consider that the motion of our cilia is understood to be the motion of the ACEO flows. That is, as shown in Fig. 6(c), the main mechanism of the up-wing motion is that the ACEO downflow at the edge produces the motion of the beam and thus lower and upper connection flows appear. Here, we use the term "connection flow" in the sense that it connects the ACEO downflow with the flows perpendicular to the upper and lower beam surfaces. As a result, we observe the up-wing motion with the edge vortex flow in the clockwise (CW) direction. Therefore, if 


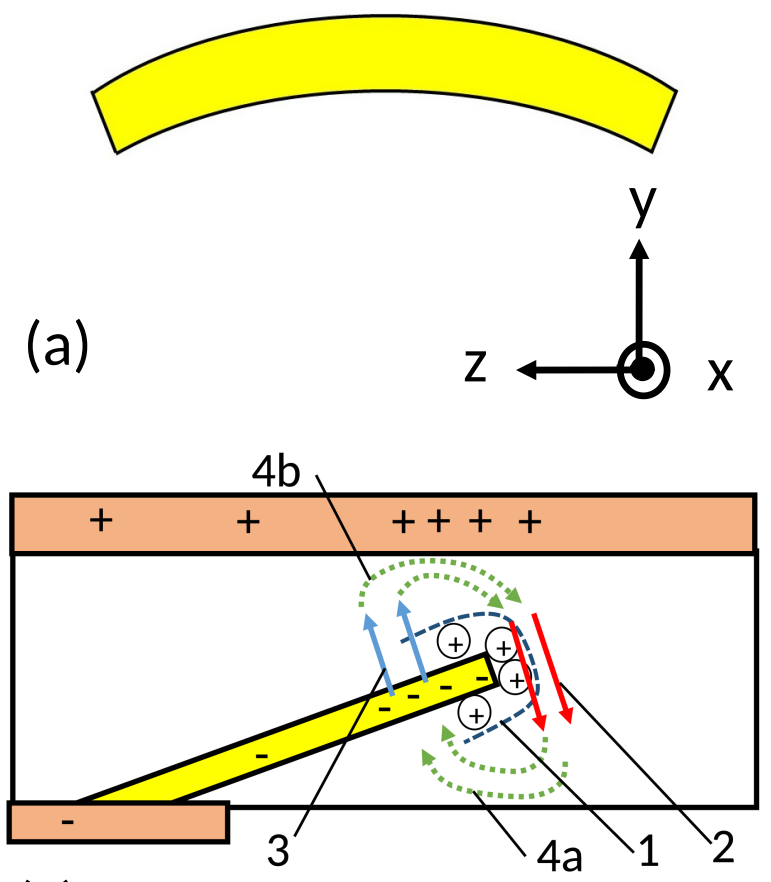

(c)
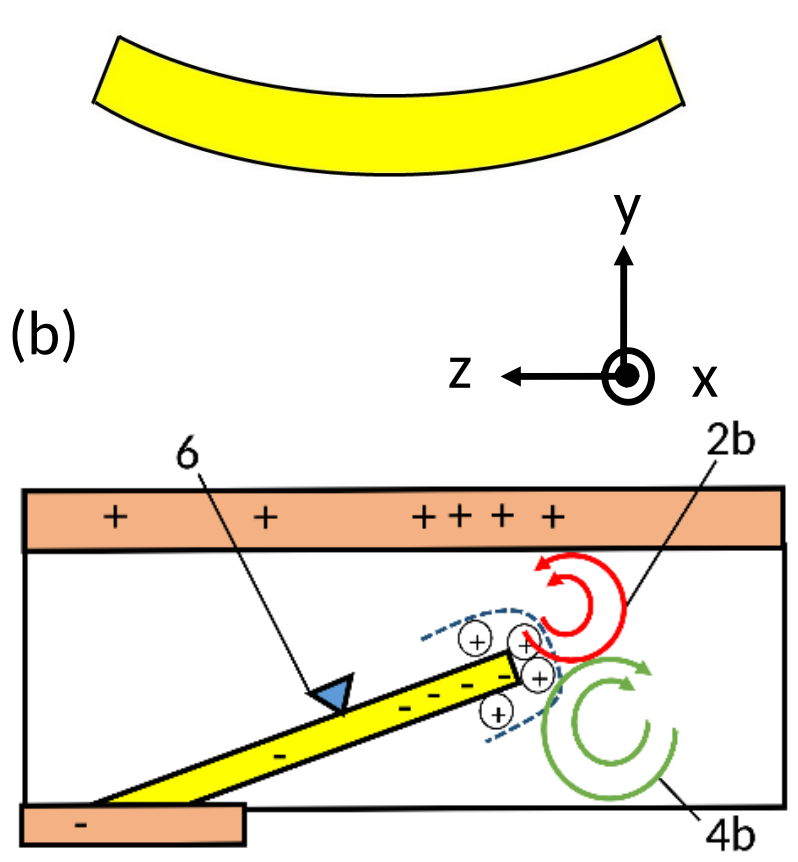

(d)

FIG. 6. (Color online) Schematic of the model for the large valving (up-wing) motion due to AC electro-osmotic flows [(c) and (d)] with the hypothetical figures of the cross-section of the beam [(a) and (b)]. 1: electro double layer, 2: ACEO flow during up-wing motion, 2b: ACEO vortex flow at the stopped state, 3: up-wing motion of the beam, 4: lower connection flow during up-wing motion, 4b: lower vortex flow at the stopped state, 5: upper connection flow during up-wing motion, and 6: conceptual or real obstacle to stop the beam.

the beam is stopped by real or conceptual obstacles, we can observe the edge vortex flow due to counterclockwise (CCW) ACEO with the vortex flow in the CW direction at the lower region, as shown in Fig. 6(d). Note that the connection flow (denoted by 4) during the up-wing motion and the lower vortex flow (denoted by $4 \mathrm{~b}$ ) at the stopped state are the bulk flows that are produced by the ACEO downflow, and they function as lift forces for the wide beam. 


\section{Possibility of another mechanism}

Although some researchers may consider that this edge vortex flow occurs not because of ACEO flow but only by the motion activated by dielectrophoresis (DEP), we can understand that the elastic beam is driven by the ACEO flow from the visualization of the flow fields. That is, when the motion of the beam was stopped by an obstacle, we observed that upper and lower vortex flows continued to move, as shown in Fig. 3(c). In Fig. 3(c), the upper vortex flow resulted from the flow understood to be the flow due to ACEO near the edge, whereas the lower vortex flow initially resulted from the inertial flow without displacement space and then being driven by the upper vortex flow after the inertial effect disappeared. Here, the inertial flow was due to the flow velocity before the flow was suddenly stopped. Thus, the upper and lower vortices continued during the pausing period, even after the inertial flow disappeared ( $\sim 0.3 \mathrm{~s}$ from the video data). In other words, the flow fields enabled us to interpret that the elastic beam was driven by the AC electro-osmotic flows, although there is insufficient evidence completely to deny a partial contribution of the DEP force to the valving motion. As discussed in several papers, it is probable that the DEP force minimally contributes to the effect $[17,18]$. However, the DEP force is the Maxwell stress that operates directly on the object and never causes flow when the object is stopped. Thus, it is reasonable to consider that the upper vortex flow that continues after the inertial flow has disappeared is the ACEO flow. In any case, we can consider that the upper vortex flow understood to be the flow due to ACEO contributes to pulling the beam up; i.e., the flow understood to be the ACEO flow pulls the beam up although the DEP force also may pull the beam up to some extent. Note that some researchers may consider that the upper edge vortex flow is caused by stopping the beam suddenly. However, the possibility is very low since we can observe the upper edge vortex even after the inertial flow disappears.

\section{Why we succeeded in observing a stable large valving motion}

As discussed in our previous paper [12], the maximum displacement of the beam is phenomenologically described by $h_{\max }=\frac{F_{A C E O L^{3}}}{3 E I} \propto \frac{L^{3}}{E d^{2}}$, where $F_{A C E O}=\frac{C_{e} W d \epsilon V_{0}^{2}}{2 \lambda_{D} h_{f}}$ is the edge force due to an ACEO flow, $I=\frac{W d^{3}}{12}$ is the second moment of area, $E$ is Young's modulus, $\lambda_{D}$ is the Debye screening length, $\epsilon$ is the permittivity of water, and $c_{e}$ is a shape factor. 
Therefore, the reason why we succeeded in observing a stable large valving motion compared to the gold leaf beam experiment $\left(E^{A u}=78 \mathrm{GPa}, d^{A u} \simeq 1 \mu \mathrm{m}\right.$, and $\left.L=4.25 \mathrm{~mm}\right)$ [11] is that we obtained a stable structure by a tenfold increase in $d$ and we increased $h_{\max }$ by an approximately 100-fold reduction in $E$ (and by increasing $L$ approximately three times) with the Au-coated PE film $\left(E^{P E}=0.4\right.$ to $1.3 \mathrm{GPa}, d^{P E} \simeq 10 \mu \mathrm{m}$, and $\left.L=15 \mathrm{~mm}\right)$. Moreover, the wide ACEO cilia have remarkable advantages for valving [8], pumping [9], and catching [10] functions than the narrow ACEO cilia demonstrated in our previous papers [11, 12], since the wide ACEO cilia control fluids more efficiently than the narrow ACEO cilia. Thus, experimental proof of the success of wide ACEO cilia is important, although the functions of both the wide and narrow ACEO cilia require further exploration in the future.

\section{E. Meaning of our study}

In this study, we have experimentally proved the large valving motion of a wide plate beam under AC electric fields, although we theoretically predicted large valving motion in our previous study [8]. In particular, the flat region in Fig. 5(c) shows that we can achieve the valving function with the wide ACEO elastic actuator, although the valving motion in the flow will have to be demonstrated experimentally in the future. By directly observing the flow field, we have shown that the motion of our cilia is understood to be the motion of the ACEO flows. This knowledge is important to establish a design base for ACEO cilia and improve ACEO cilia in the future. We also found that a wide beam moves in the upper direction largely because of the edge vortex flow understood to be the flow due to ACEO and the incoming lift flow into the bottom region of the valve.

\section{CONCLUSION}

In summary, we have shown that a wide Au-coated plastic-film beam displays large valving motion in water under $\mathrm{AC}$ electric fields. In particular, by the direct observation of the flow fields during the up-wing motion, we have shown that the large valving motion of the wide Au-coated beam is understood to be the motion due to the $\mathrm{AC}$ osmotic flows at the edge of the beam, and we believe that our findings contribute significantly to realize 
wide beam artificial cilia using ACEO.

[1] J. den Toonder and P. Onck, Trends Biotechnol. 31, 85 (2013).

[2] T. Squires and S. Quake, Rev.Mod.Phys. 77, 977 (2005).

[3] S. Hanasoge, P. Hesketh, and A. Alexeev, Microsystems Nanoengineering 4, 11 (2018).

[4] B. Panigrahi, C. Lu, N. Ghayal, and C. Chen, Scientific Reports 8, 4605 (2018).

[5] M. Asadnia, K. K. A.G.P. Kottapalli, M. Warkiani, J. Miao, D. Corey, and M. Triantafyllou, Scientific Reports 6, 32955 (2016).

[6] K. Mani, T. Chang, B. Panigrahi, and C. Chen, Scientific Reports 6, 36385 (2016).

[7] J. d. Toonder, F. Bos, D. Broer, L. Filippini, M. Gillies, J. de Goede, T. Mol, M. Reijme, W. Talen, H. Wilderbeek, V. Khatavkar, and P. Anderson, Lab ChipArtificial cilia for active micro-fluidic mixing 8, 533 (2008).

[8] H. Sugioka, Phys. Rev. Applied 3, 064001 (2015).

[9] H. Sugioka, Journal of the Physical Society of Japan 85, 104001 (2016).

[10] H. Sugioka, Journal of the Physical Society of Japan 86, 014401 (2017).

[11] H. Sugioka and N. Nakano, Phys. Rev. E 97, 013105 (2018).

[12] H. Sugioka, N. Nakano, and Y. Mizuno, Journal of the Physical Society of Japan 88, 084801 (2019).

[13] A. Ramos, H. Morgan, N. G. Green, and A. Castellanos, J. Colloid Interf. Sci. 217, 420 (1999).

[14] M. Z. Bazant and T. M. Squires, Phys. Rev. Lett. 92, 066101 (2004).

[15] N. G. Green, A. Ramos, A. González, H. Morgan, and A. Castellanos, Phys. Rev. E 61, 4011 (2000).

[16] A. Ramos, A. Gonzalez, A. Castellanos, N. G. Green, and H. Morgan, Phys. Rev. E 67, $056302(2003)$.

[17] T. M. Squires and M. Z. Bazant, J. Fluid Mech. 560, 65 (2006).

[18] H. Sugioka, Phys. Rev. E 81, 036301 (2010). 